\title{
One-particle spectroscopic intensities as a signature of shape phase transition: The $\boldsymbol{\gamma}$-unstable case
}

\author{
C. E. Alonso, ${ }^{1}$ J. M. Arias, ${ }^{1}$ and A. Vitturi ${ }^{1,2}$ \\ ${ }^{1}$ Departamento de Física Atómica, Molecular y Nuclear, Facultad de Física, Universidad de Sevilla, \\ Apartado 1065, 41080 Sevilla, Spain \\ ${ }^{2}$ Dipartimento di Fisica Galileo Galilei and INFN, Via Marzolo 8, 35131 Padova, Italy
}

(Received 27 April 2006; published 3 August 2006)

\begin{abstract}
We investigate the evolution of one-particle spectroscopic intensities as a possible signature of shape phase transitions. The study describes the odd systems in terms of the interacting boson-fermion model. We consider the particular case of an odd $j=3 / 2$ particle coupled to an even-even boson core that undergoes a phase transition from spherical $\mathrm{U}(5)$ to $\gamma$-unstable $\mathrm{O}(6)$ situation. At the critical point, our findings are compared with the one-particle spectroscopic intensities that can be obtained within the $\mathrm{E}(5 / 4)$ model proposed by [F. Iachello, Phys. Rev. Lett. 95, 052503 (2005); F. Iachello, in Symmetries and Low-Energy Phase Transitions in Nuclear Structure Physics, edited by G. Lo Bianco (University of Camerino Press, Camerino, Italy, in press)].
\end{abstract}

DOI: 10.1103/PhysRevC.74.027301

The study of phase transitions and the concept of critical point symmetry in nuclear systems was recently extended to the case of odd nuclei. In particular, in the case of an odd $j=3 / 2$ particle coupled to an even-even boson core that undergoes a phase transition from spherical U(5) to $\gamma$-unstable $\mathrm{O}(6)$ situation, the occurrence of the $\mathrm{E}(5 / 4)$ critical point symmetry was proposed by Iachello [1,2]. The same problem was attacked by the authors in the framework of the interacting boson-fermion model [3]. Recently ${ }^{135} \mathrm{Ba}$ was proposed as the first experimental example of a Bose-Fermi critical point symmmetry E(5/4) [4].

A critical issue in the description of phase transitions is obviously the proper individuation of the critical points as a function in the control parameters. In our case this can be done, for example, by looking at the sudden change in the form of the energy surfaces associated with the intrinsic fermion-boson state. Other indications of the transition come from the rapid variation of other quantities, such as ratios of particular level energies or values of particular electromagnetic transitions. Because the phase transition involves both the even and the odd systems, in this brief report we examine the evolution of the matrix elements connecting precisely the even and the odd nuclei, namely, the one-particle transfer spectroscopic intensities.

In the framework of the interacting boson-fermion model (IBFM) [5] the coupling of a single fermion to the even-even bosonic core is described by the Hamiltonian

$$
H=H_{B}+H_{F}+V_{B F},
$$

where the term $V_{B F}$ couples the bosonic and fermionic parts. For the bosonic part, we use parametrized Hamiltonians that produce transitions between spherical and $\gamma$-unstable shapes. In particular, we use the sets of Hamiltonians [6]

$$
H_{B}^{1}=x \hat{n}_{d}-\frac{1-x}{N} \hat{Q}_{B} \cdot \hat{Q}_{B},
$$

and

$$
H_{B}^{2}=x \hat{n}_{d}+\frac{1-x}{N-1} \hat{P}_{B}^{\dagger} \cdot \hat{P}_{B}
$$

PACS number(s): 21.60.Fw, 21.60.Ev, 21.10.Jx

$$
\begin{aligned}
= & x \hat{n}_{d}+\frac{1-x}{N-1}\left(\frac{1}{4} N(N+4)-\frac{1}{4} \hat{Q}_{B} \cdot \hat{Q}_{B}\right. \\
& \left.-\frac{1}{20} \hat{L}_{B} \cdot \hat{L}_{B}-\frac{1}{2} \hat{T}_{B}^{3} \cdot \hat{T}_{B}^{3}\right) .
\end{aligned}
$$

The operators in the Hamiltonians are given by

$$
\begin{aligned}
\hat{n}_{d} & =\sum_{\mu} d_{\mu}^{\dagger} d_{\mu}, \\
\hat{P}_{B}^{\dagger} & =\frac{1}{2}\left(d^{\dagger} \cdot d^{\dagger}-s^{\dagger} \cdot s^{\dagger}\right), \\
\hat{L}_{B} & =\sqrt{10}\left(d^{\dagger} \times \tilde{d}\right)^{(1)}, \\
\hat{Q}_{B} & =\left(s^{\dagger} \times \tilde{d}+d^{\dagger} \times \tilde{s}\right)^{(2)}, \\
\hat{T}_{B}^{3} & =\left(d^{\dagger} \times \tilde{d}\right)^{(3)},
\end{aligned}
$$

and $N$ is the total number of bosons. Both Hamiltonians produce, varying the parameter $x$ from 1 to 0 , a transition between the two extreme situations characteristic of $U(5)$ and $\mathrm{O}(6)$ symmetries: at $x_{c}=(4 N-8) /(5 N-8)$ for the first case and at $x_{c}=1 / 2$ for the second. The two paths connecting $\mathrm{U}(5)$ and $\mathrm{O}(6)$ are however different and can, for example, be viewed as two different lines in the nuclear phase diagram (known as Casten triangle [7]), with the respective critical point lying in two different points in the triangle. Note that for any value of $x$ both these Hamiltonians maintain the degeneracies typical of the $\mathrm{O}(5)$ symmetry. Consistently with this, within the IBM coherent state formalism [8-10], these Hamiltonians always produce an energy surface that is independent of the $\gamma$ degree of freedom. In the $\beta$ variable, the energy surfaces display a spherical minimum in $\beta=0$ for $x$ larger than the critical value, while having a deformed minimum for values of $x$ smaller than the critical values. At the critical point, the energy surface in both cases acquires in leading order a $\beta^{4}$ behavior [6], but differs for the higher order terms. For a finite number of bosons, therefore, the two Hamiltonians are 
expected to give very similar energy spectra, but with some quantitative differences.

With the boson part given by either Hamiltonian (2) or (3), for the fermion and boson-fermion parts we take the particular choice of a particle moving in a single $j$ shell $j=3 / 2$. The coupling term is assumed to be of the form

$$
V_{B F}^{1}=-2 \frac{1-x}{N} \hat{Q}_{B} \cdot \hat{q}_{F},
$$

with the boson Hamiltonian $H_{B}^{1}$ and

$$
V_{B F}^{2}=-\frac{1-x}{4(N-1)}\left(2 \hat{Q}_{B} \cdot \hat{q}_{F}+\frac{2}{5} \hat{L}_{B} \cdot \hat{l}_{F}+4 \hat{T}_{B}^{3} \cdot \hat{t}_{F}^{3}\right),
$$

in the case of the boson Hamiltonian $H_{B}^{2}$. The fermion operators are defined as $\hat{l}_{F}=-\sqrt{5}\left(a_{3 / 2}^{\dagger} \times \tilde{a}_{3 / 2}\right)^{(1)}, \hat{q}_{F}=\left(a_{3 / 2}^{\dagger} \times\right.$ $\left.\tilde{a}_{3 / 2}\right)^{(2)}$, and $\hat{t}_{F}^{3}=(1 / \sqrt{2})\left(a_{3 / 2}^{\dagger} \times \tilde{a}_{3 / 2}\right)^{(3)}$. These choices of the fermion space and the boson-fermion interactions are such that for both sets of Hamiltonians one recovers, in the extreme cases, the Bose-Fermi symmetry [5] associated with the $\operatorname{Spin}^{B F}(6)$ group (for $x=0$ ) and the vibrational $\mathrm{U}^{B}(5) \otimes \mathrm{SU}^{F}(4)$ case (for $x=1$ ). In analogy with the overall $\mathrm{O}(5)$ structure in the even case, this selection guarantees the preservation of the degeneracies associated with the $\operatorname{Spin}^{B F}(5)$ symmetry for any value of $x$. Our states are characterized by the quantum numbers $\tau_{1}, \tau_{2}, J$. We also characterize the different bands with their asymptotic $\sigma_{1}$ value, with the caution that it is a good quantum number only in the $\operatorname{Spin}^{B F}(6)$ limit.

The two resulting spectra for the two Hamiltonians at the respective critical points are shown in Figs. 1(a) and 1(b). As expected the two spectra are quite similar, but differences can be appreciated. In particular differences

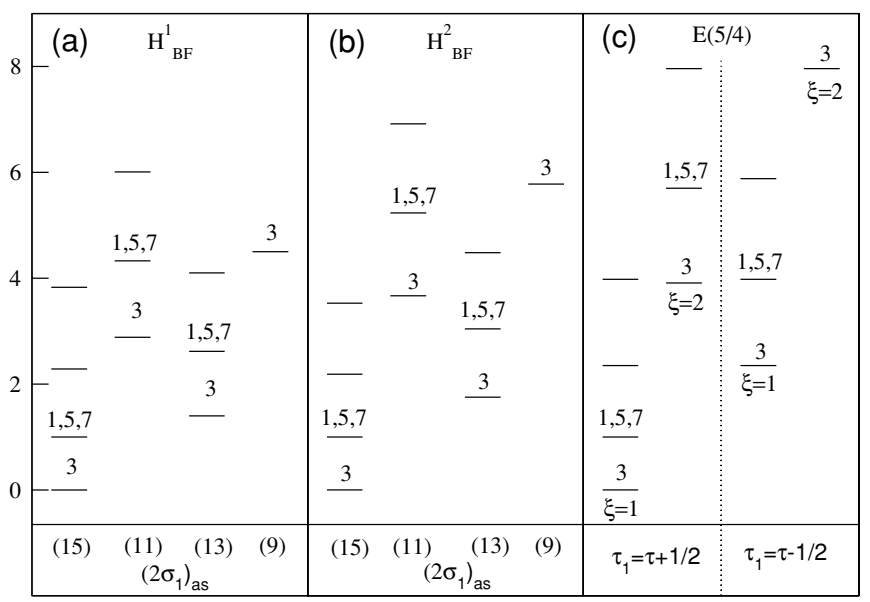

FIG. 1. Energy levels (normalized to the energy of the first excited state). (a,b) Results of the IBFM for the two Hamiltonians $H_{B F}^{1}$ and $H_{B F}^{2}$ at the respective critical points. A number $N=7$ of bosons is assumed. For the lowest levels twice the angular momenta are given; for the other degenerate levels we refer for example to Ref. [5]. To characterize the different bands we quote twice the asymptotic quantum number $\sigma_{1}$. (c) Corresponding predictions for the $\mathrm{E}(5 / 4)$ model. Each band is characterized by the class $\left(\tau_{1}=\tau+1 / 2\right.$ or $\tau_{1}=\tau-1 / 2$ ) and by the $\xi$ quantum number.
TABLE I. $B(E 2)$ values predicted for the indicated transitions within the IBFM with $H_{B F}^{1}$ and $H_{B F}^{2}$ Hamiltonians and within the $\mathrm{E}(5 / 4)$ critical symmetry (in this case only the boson part of the transition operator is included [1,2]). States are labeled by the "asymptotic" quantum number $\sigma_{1}$ and by the good $\tau_{1}, \tau_{2}, J$ quantum numbers.

\begin{tabular}{cccc}
\hline \hline & $H_{B F}^{1}$ & $H_{B F}^{2}$ & $E(5 / 4)$ \\
\hline$\left[\frac{15}{2}, \frac{3}{2}, \frac{1}{2}, \frac{7}{2}\right] \rightarrow\left[\frac{15}{2}, \frac{1}{2}, \frac{1}{2}, \frac{3}{2}\right]$ & 100 & 100 & 100 \\
{$\left[\frac{15}{2}, \frac{5}{2}, \frac{1}{2}, \frac{11}{2}\right] \rightarrow\left[\frac{15}{2}, \frac{3}{2}, \frac{1}{2}, \frac{7}{2}\right]$} & 141 & 145 & 164 \\
{$\left[\frac{13}{2}, \frac{1}{2}, \frac{1}{2}, \frac{3}{2}\right] \rightarrow\left[\frac{15}{2}, \frac{1}{2}, \frac{1}{2}, \frac{3}{2}\right]$} & 23 & 33 & 101 \\
{$\left[\frac{11}{2}, \frac{1}{2}, \frac{1}{2}, \frac{3}{2}\right] \rightarrow\left[\frac{15}{2}, \frac{1}{2}, \frac{1}{2}, \frac{3}{2}\right]$} & 0.05 & 0.04 & 0 \\
\hline \hline
\end{tabular}

are present in the positions of the different bands. Agreement of the same quality is also obtained for the $B(E 2)$ transition probabilities. The values of some selected transitions are given in Table I. We have chosen (assuming as 100 the inband transition $\left[\sigma_{1}=15 / 2, \tau_{1}=3 / 2\right.$, $\left.\left.\tau_{2}=1 / 2, J=7 / 2\right] \rightarrow[15 / 2,1 / 2,1 / 2,3 / 2]\right)$ the inband transition $\quad[15 / 2,5 / 2,1 / 2,11 / 2] \rightarrow[15 / 2,3 / 2,1 / 2,7 / 2]$ and the intraband transitions $[13 / 2,1 / 2,1 / 2,3 / 2] \rightarrow[15 / 2$, $1 / 2,1 / 2,3 / 2]$ and $[11 / 2,1 / 2,1 / 2,3 / 2] \rightarrow[15 / 2,1 / 2,1 / 2$, $3 / 2]$. For the transition operator we have assumed for the IBFM calculations $T^{E 2}=\hat{Q}_{B}+\hat{q}_{F}$ to be consistent with the choice in the Hamiltonian. For comparison, in Fig. 1(c) and the third column of Table I, we also show the corresponding predictions for energies and transitions within the $E(5 / 4)$ critical point symmetry. As already discussed in Ref. [6] for the even case and in Ref. [3] for the odd case, the spectra obtained within the IBM/IBFM approach are rather similar to those obtained within the $\mathrm{E}(5)$ and $\mathrm{E}(5 / 4)$ critical point symmetries. Some quantitative differences arise for the relative positions of the different bands, for example, in the position of the $0^{+}$bandhead of the first excited band in the even case or the first excited band (with $J=3 / 2$ bandhead) in the odd case. Levels in the excited bands in the IBFM are also somewhat compressed with respect to the $\mathrm{E}(5 / 4)$ case. All these differences can be traced back to the use in these cases of an infinite square well potential in the $\beta$ variable, at variance with the dominant $\beta^{4}$ dependence in the IBM/IBFM cases.

For a useful comparison, it is necessary to have a way of properly connecting our states with the two classes of states present in $\mathrm{E}(5 / 4)$, with either $\tau_{1}=\tau+1 / 2$ or $\tau_{1}=\tau-1 / 2$. A hint can come from the analysis of the IBFM wave functions. The ground-state wave function has a large overlap $(84 \%$ in $H_{B F}^{1}$ and $88 \%$ in $\left.H_{B F}^{2}\right)$ with the $\left(0_{1}^{+} \times 3 / 2\right)$ and is therefore naturally associated with the lowest $\left(\tau=0, \tau_{1}=1 / 2\right)$ state. Our second $3 / 2$ state has a large overlap (83\% in $H_{B F}^{1}$ and $87 \%$ in $\left.H_{B F}^{2}\right)$ with the $\left(2_{1}^{+} \times 3 / 2\right)$ and must therefore correspond to the lowest $\left(\tau=1, \tau_{1}=1 / 2\right)$ state. Similarly the 4 th $J=3 / 2$ state (3rd bandhead) in $H_{B F}^{1}$ (and the 5th in $H_{B F}^{2}$ ) has large overlaps $\left(92 \%\right.$ in $H_{B F}^{1}$ and $94 \%$ in $\left.H_{B F}^{2}\right)$ with the $\left(0_{2}^{+} \times 3 / 2\right)$, so it can be put in correspondence with the $\left(\xi=2, \tau=0, \tau_{1}=\right.$ $1 / 2$ state). Note that, at variance with the $\mathrm{E}(5 / 4)$ case, our states have only approximately good values of $\tau$, but we have noticed 


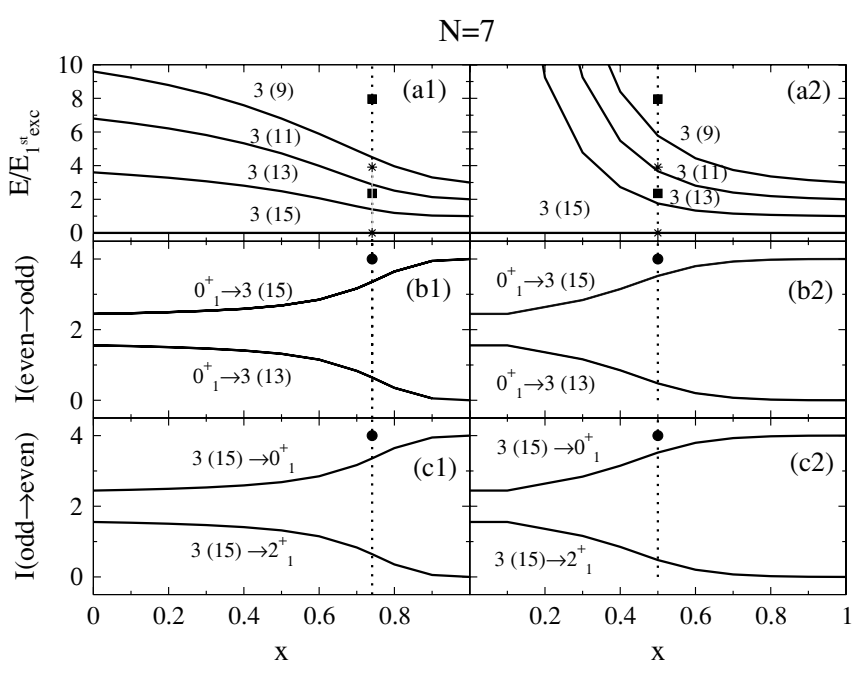

FIG. 2. In the upper panels the energies (normalized to the energy of the first excited $\left[\tau_{1}=3 / 2, \tau_{2}=1 / 2, J=(1 / 2,5 / 2,7 / 2)\right]$ multiplet) of the different $\left[\tau_{1}=1 / 2, \tau_{2}=1 / 2, J=3 / 2\right]$ states are displayed as a function of the parameter $x$. Each state is labeled according to the asymptotic $(x=0)$ value of the quantum number $\sigma_{1}$. The two columns refer to the results obtained with the $H_{B F}^{1}$ (left) and $H_{B F}^{2}$ (right) Hamiltonians. In both cases the dashed vertical line indicates the position of the critical point. For that value we indicate as stars and squares the values of the analogous levels in the $\mathrm{E}(5 / 4)$ model with $\tau_{1}=\tau+1 / 2$ and $\tau_{1}=\tau-1 / 2$, respectively. In the second (third) row we plot the square of the reduced matrix element of the one-fermion creation (annihilation) operator from the even to odd (and odd to even) system as a function of the parameter $x$. In the former case we start from the ground state of the even system and consider final $\left[\tau_{1}=1 / 2, \tau_{2}=1 / 2, J=3 / 2\right]$ states. In the latter case we start from the ground state of the odd nucleus and populate possible $L=0,2$ states. At the critical point, the circle corresponds to the only allowed transition.

that the states become progressively purer as we increase the number of bosons.

The full behavior of the energy levels with the parameter $x$ along the transition from $\mathrm{U}^{B}(5) \otimes \mathrm{SU}^{F}(4)$ to $\operatorname{Spin}^{B F}(6)$ is displayed in the upper panels of Fig. 2 for both choices of the Hamiltonian. We have selected here only the $J=3 / 2$ states with $\tau_{1}=1 / 2$, because they are the only ones that can be fed by the one-particle creation operator from the ground state of the even nucleus. In correspondence with critical values of the parameter $x$ we mark as stars and squares the energies of the analogous levels in the $\mathrm{E}(5 / 4)$ model with $\tau_{1}=\tau+1 / 2$ and $\tau_{1}=\tau-1 / 2$, respectively.

As mentioned, very recently the first experimental measurements to test the $\mathrm{E}(5 / 4)$ predictions for excitation energies and E2 transitions have been presented in connection to the nucleus ${ }^{135} \mathrm{Ba}$ [4]. In this reference a comparison with an IBFM fit is also presented. It is worth noting that our calculations are not fits, but just the results obtained at the critical point for specific Hamiltonians for $N=7$ (which is not the boson number of $\left.{ }^{135} \mathrm{Ba}\right)$. In comparison with experimental data, it should be noted that in our calculation the only single-particle level included is the $d_{3 / 2}$ as in $\mathrm{E}(5 / 4)$; consequently, states coming from $s_{1 / 2}$, observed in experiments, are absent in our results.

A question addressed in Ref. [4] is the possibility of getting the splitting of the first excited multiplet, as it is observed in ${ }^{135} \mathrm{Ba}$. In our calculations we have the possibility of adding to the Hamiltonian a term of the type $C_{2}\left(\operatorname{Spin}^{B F} 3\right)$ without loosing the $\gamma$ unstability. This addition will split this multiplet, but the energies will vary monotonically with spin, inconsistent with the data for ${ }^{135} \mathrm{Ba}$. The addition of an exchange interaction will explain the observed splitting, but it will break the $\operatorname{Spin}^{B F}(5)$ symmetry that we have along the transition from $\mathrm{U}^{B}(5) \otimes \mathrm{SU}^{F}(4)$ to $\mathrm{O}^{B}(6) \otimes \mathrm{SU}^{F}(4)$.

We come now to the one-fermion addition (removal) matrix elements $\left\langle f\left\|a_{3 / 2}^{\dagger}\left(\tilde{a}_{3 / 2}\right)\right\| i\right\rangle$. In leading order the square of these matrix elements will give the one-particle-transfer spectroscopic intensities for stripping and pick-up reactions [5]. In the former case we start from the ground state of the even nucleus adding a fermion, with the possibility therefore of populating only states with $J=3 / 2$ and $\tau_{1}=1 / 2$ (the transfer operator chosen is $\left.a_{3 / 2}^{\dagger}\right)$. In the latter case, we start from the $\left(J=3 / 2, \tau_{1}=1 / 2\right)$ ground state of the odd nucleus and remove a fermion with the operator $\tilde{a}_{3 / 2}$, populating therefore only states with $L=0^{+}, 2^{+}$and $\tau=0,1$. The even-to-odd and odd-to-even squared reduced matrix elements are given in the second and third rows of Fig. 2 as a function of the parameter $x$ for the two choices of Hamiltonians. Again the two Hamiltonian give rather similar results. In correspondence to the phase transition the spectroscopic intensities show a characteristic change of behavior from the spherical case $(x=1)$, where only ground-to-ground transitions are allowed, to the $(\gamma$-unstable) deformed case $(x=0)$, where transitions to excited states are allowed.

At the critical point, we can compare the IBM/IBFM results with the predictions of the $E(5) / E(5 / 4)$ models. This can be done by calculating one-fermion matrix elements using the explicit wave functions of the different states in the even $\mathrm{E}(5)$ and odd $\mathrm{E}(5 / 4)$ models in the variables $(\beta, \gamma$, and Euler angles $\left.\theta_{i}\right)$. These matrix elements give orthogonality conditions in the $\gamma$ and $\theta_{i}$ integration, reducing to just one-dimensional integrals in the $\beta$ variable. In particular, starting from the ground state of the even core $(\xi=1, \tau=0)$ we will only populate final states in the odd with $\xi^{\prime}, \tau=0, \tau_{1}=1 / 2, J=3 / 2$ states of the odd, with matrix elements

$$
\begin{gathered}
\left\langle\xi^{\prime}, \tau=0, \tau_{1}=1 / 2, \tau_{2}=1 / 2, J=3 / 2\left\|a_{3 / 2}^{\dagger}\right\| \xi=1,\right. \\
\left.\tau=0, L=0^{+}\right\rangle=-\sqrt{\frac{2 J+1}{2 L+1}} \int_{0}^{\beta_{w}} f_{1,0}(\beta) F_{\xi^{\prime}, 0,1 / 2,1 / 2}(\beta) \beta^{4} d \beta .
\end{gathered}
$$

The function $f_{\xi, \tau}(\beta)=c_{\xi, \tau} \beta^{-3 / 2} J_{v}\left(x_{\xi, \tau} \beta / \beta_{w}\right)$ is associated with the states of the boson part. The quantity $x_{\xi, \tau}$ is the $\xi$ zero of $J_{v}(z)$ with $v=\tau+3 / 2, \beta_{w}$ is the dimension of the infinite square well in $\beta$, and $c_{\xi, \tau}$ is a normalization constant. The corresponding function $F_{\xi^{\prime}, \tau, \tau_{1}, \tau_{2}}(\beta)$ describes the $\beta$ part of the wave functions in the fermion case and is given by $F_{\xi^{\prime}, \tau, \tau_{1}, \tau_{2}}(\beta)=c_{\xi^{\prime}, \tau, \tau_{1}, \tau_{2}} \beta^{-3 / 2} J_{v^{\prime}}\left(x_{\xi^{\prime}, \tau, \tau_{1}, \tau_{2}} \beta / \beta_{w}\right)$, where $x_{\xi^{\prime}, \tau, \tau_{1}, \tau_{2}}$ is the $\xi^{\prime}$-th zero of $J_{v^{\prime}}(z)$ with $v^{\prime}=$ $\sqrt{2 \tau(\tau+3)-\tau_{1}\left(\tau_{1}+3\right)-\tau_{2}\left(\tau_{2}+1\right)+19 / 4}$. In the partic- 
ular case of $\tau=0, \tau_{1}=1 / 2$, and $\tau_{2}=1 / 2$, both $v$ and $v^{\prime}$ get the same value $3 / 2$ and the functions $f_{\xi, 0}(\beta)$ and $F_{\xi^{\prime}, 0,1 / 2,1 / 2}(\beta)$ become identical. So, because of the orthogonality condition, starting from the $\xi=1$ ground state in the even nucleus we will only have connecting matrix elements to the $\xi^{\prime}=1$ state in the odd, namely the ground state, with vanishing matrix elements to all other states. All the transfer strength, therefore, will go into the ground state.

A similar situation occurs for the matrix elements connecting the ground state of the odd nucleus with the different states in the even system. The orthogonality condition in the $\left(\gamma, \theta_{i}\right)$ variables implies that we can only populate states with $\tau=0$, therefore, excluding, for example, the first $2^{+}$state which is characterized by $\tau=1$. But in addition, in this particular case, the equality of the $\beta$ wave functions for the ground states in even and odd systems allows only a transition to the $\xi=1, \tau=0$, and again all transfer strength will be concentrated in the ground state.

To summarize, we show that one-particle spectroscopic intensities between neighbor even and odd nuclei can be used as a signature of shape phase transitions, in addition to the normally used energies and electromagnetic matrix elements. We illustrate this point by considering within the interacting boson-fermion model the simple case of the coupling of a $j=3 / 2$ particle to an even core undergoing a transition from spherical $\mathrm{U}(5)$ to $\gamma$-unstable $\mathrm{O}(6)$ situation. Different options for the boson Hamiltonian describing the transition are considered. At the critical point, our results for the one-particle spectroscopic intensities are compared with the results obtained within the $\mathrm{E}(5 / 4)$ critical point symmetries. Spectroscropic intensities are shown to help in clarifying similarities and differences between IBFM and $\mathrm{E}(5 / 4)$ approaches.

This work was partially supported the Spanish Ministerio de Educación y Ciencia and the European Regional Development Fund (FEDER) under Project FIS2005-01105 and by INFN. Andrea Vitturi acknowledges financial support from SEUI (Spanish Ministerio de Educación y Ciencia) for a sabbatical year at the University of Sevilla.
[1] F. Iachello, Phys. Rev. Lett. 95, 052503 (2005).

[2] F. Iachello, in Symmetries and Low-Energy Phase Transitions in Nuclear Structure Physics, edited by G. Lo Bianco (University of Camerino Press, Camerino, Italy, in press).

[3] C. E. Alonso, J. M. Arias, L. Fortunato, and A. Vitturi, Phys. Rev. C 72, 061302(R) (2005).

[4] M. S. Fetea et al., Phys. Rev. C 73, 051301(R) (2006).

[5] F. Iachello and P. Van Isacker, The Interacting Boson-Fermion Model (Cambridge University Press, Cambridge, UK, 1991).
[6] J. M. Arias, C. E. Alonso, A. Vitturi, J. E. García-Ramos, J. Dukelsky, and A. Frank, Phys. Rev. C 68, 041302(R) (2003).

[7] R. F. Casten and J. Cizewski, Nucl. Phys. A309, 477 (1978).

[8] J. N. Ginocchio and M. W. Kirson, Nucl. Phys. A350, 31 (1980).

[9] A. E. L. Dieperink, O. Scholten, and F. Iachello, Phys. Rev. Lett. 44, 1747 (1980).

[10] A. Bohr and B. R. Mottelson, Phys. Scr. 22, 468 (1980). 